\title{
JUVENTUD, MIGRACIÓN Y DISCRIMINACIÓN EN EL CHILE CONTEMPORÁNEO
}

\author{
ANDREA ARAVENA REYES* \\ CAROLINA AlT ÁlVAREZ**
}

\begin{abstract}
RESUMEN
En este trabajo se analiza el tema de la inmigración juvenil en el Chile Contemporáneo y se aborda la percepción desde «lo juvenil» de la alteridad en el país, tanto desde el punto de vista de la población chilena como desde el punto de vista de los inmigrantes. Lo anterior, a partir de fuentes de información cuantitativas, como el Censo del año 2002 y la CASEN 2006, las versiones Quinta y Sexta de la serie de Encuestas de Juventud de Chile (2007 y 2009), y de un conjunto de antecedentes cualitativos de producción propia, como resultados de grupos de discusión realizados a inmigrantes jóvenes en Chile, ${ }^{1}$ resultados de entrevistas en profundidad a inmigrantes peruanos, ${ }^{2}$ y de grupos de discusión con personas chilenas sobre inmigrantes. ${ }^{3}$
\end{abstract}

\section{PALABRAS CLAVE: JUVENTUD, MIGRACIÓN, DISCRIMINACIÓN, IMAGINARIOS SOCIALES, PERUANOS}

* Antropóloga, Doctora en Antropología, EHESS, París. Docente de la Universidad de Concepción. E-Mail: andrea.aravena@udec.cl.

** Socióloga, en curso Magíster en Gestión y Políticas Públicas, Universidad de Chile. Investigadora del Departamento de Planificación y Estudios del INJUV. E-Mail: calt@injuv.gob.cl.

Artículo presentado en el VI Congreso Chileno de Sociología y Encuentro Pre Alas Chile 2011.

1 Resultados de 16 Grupos de Discusión a inmigrantes jóvenes de distintas nacionalidades en todo el país, elaborados en el marco de la investigación: «Jóvenes migrantes: inclusión social y desafíos para las políticas públicas en juventud», elaborado por Corporación Centro de Análisis de Políticas Públicas, INJUV (2011) del cual Carolina Alt fue investigadora.

2 Opiniones resultantes de 18 entrevistas en profundidad a inmigrantes peruanos en Santiago y Concepción, entre los años 2010 y 2011, en el marco del proyecto Fondecyt N ${ }^{\mathrm{o}} 1100928$ (2010-2012), del cual Andrea Aravena es coinvestigadora.

3 Resultados de 20 Grupos de Discusión realizados a chilenos en Valparaíso y Santiago, entre los años 2008 y 2009, en el marco del proyecto Fondecyt No1071090 (2007-2009), del cual Andrea Aravena fue coinvestigadora. 


\title{
JUVENTUDE, MIGRAÇÃO E DISCRIMINAÇÃO NO CHILE CONTEMPORÂNEO
}

\begin{abstract}
RESUMO
Neste trabalho, analisa-se o tema da imigração juvenil no Chile contemporâneo, abordando a percepção a partir do «juvenil», da alteridade no país, tanto do ponto de vista da população chilena como do ponto de vista dos imigrantes. Utilizam-se fontes de informação quantitativas, como o Censo de 2002 e a CASEN 2006, as versões Quinta e Sexta da série de Levantamentos de Juventude do Chile (2007 e 2010), bem como um conjunto de antecedentes qualitativos de produção própria, como resultados de grupos de discussão realizados com imigrantes jovens no Chile, resultados de entrevistas em profundidade com imigrantes peruanos e de grupos de discussão com pessoas chilenas sobre imigrantes.
\end{abstract}

\author{
PALAVRAS CHAVE: JUVENTUDE, MIGRAÇÃO, DISCRIMINAÇÃO, \\ IMAGINÁRIOS SOCIAIS, PERUANOS
}

\section{YOUTH MIGRATION AND DISCRIMINATION IN CONTEMPORARY CHILE}

\begin{abstract}
This article will examine the issue of youth migration in Contemporary Chile and addresses the perception from «the youth» of otherness in the country, both from the point of view of the Chilean population and from the point of view immigrants. From sources of quantitative information, such as Census 2002 and CASEN 2006, Fifth and Sixth Series versions of the Youth Survey of Chile (2007 and 2010), and a set of qualitative background own production, as a result of discussion groups for young immigrants in Chile, results of in depth interviews with Peruvian immigrants and focus groups with people on Chilean immigrants.
\end{abstract}

KEY WORDS: YOUTH, MIGRATION, DISCRIMINATION, SOCIAL IMAGERIES, PERUVIANS 


\section{ANTECEDENTES}

LAS TRANSFORMACIONES MIGRATORIAS son procesos demográficos transversales que poseen diversas particularidades que influyen e impactan en esferas como la política, la economía, la cultura y lo social, entre otras. Es por ello que los movimientos migratorios se transforman en un fenómeno relevante a escala mundial y Sudamérica no está al margen de dicho proceso.

En Chile en los últimos años se ha presentado un incremento sustantivo y sostenido en la cantidad de personas que entran al país. La población extranjera presente en el territorio chileno se ha mantenido en rangos bajos, no superando en ningún momento de su historia el $4,5 \%$ del total de la población. Aun cuando estos datos no permiten sostener que Chile se ha convertido en un país de inmigrantes, las cifras censales nacionales, desde 1952, señalan que el país ha llegado a una magnitud absoluta de inmigrantes superior a cualquier otra época de su historia (Martínez, 2003).

El año 2002, el Censo Nacional de Población y Vivienda mostró que un $1,2 \%$ de la población total del país eran inmigrantes extranjeros residentes en Chile, correspondientes a 184.464 personas. De éstos, el 67,9\% del total de los extranjeros residentes en Chile proviene de países de América del Sur, siendo 48.176 argentinos, 37.860 peruanos, 10.919 bolivianos, 9.393 ecuatorianos, 6.895 brasileños, 4.338 venezolanos, 4.095 colombianos, 2.241 uruguayos, 1.222 paraguayos y 22 de otros países.

Según el Censo 2002, la región con mayor concentración de extranjeros es la Región Metropolitana con 112.809 individuos que representan el $61 \%$ de la población total de inmigrantes. Otras regiones que poseen un elevado número de inmigrantes son la Quinta Región con 15.948 individuos, representando el 9\% de la población total de extranjeros, seguida por la Primera Región con 12.812 personas $(7 \%)$ y la Décima Región con 8.259 inmigrantes (4\%). De la población total de inmigrantes, 61.399 corresponden a jóvenes de 15 a 29 años, de los cuales 38.837 habitan en la Región Metropolitana.

Ello nos lleva a indagar, en primer lugar, en la caracterización de la juventud inmigrante según su origen; en segundo lugar, en la construcción imaginario social dominante del inmigrante en la sociedad chilena y; en tercer lugar, en las vivencias de dichos jóvenes inmigrantes, particularmente peruanos. 


\section{CARACTERIZACión DE LA MigRACión JUVENIL EN CHILE}

De la población total de 185.000 personas nacidas en el extranjero y residentes en Chile (INE, 2002), 61.129 son jóvenes de 15 a 29 años, con una mayor proporción de mujeres (59\%) que de hombres (Casen 2006). La mayoría reside en la Región Metropolitana ${ }^{4}$ (39.057 personas, equivalente al 64,9\%), seguida de la zona centro (V, VI, VII y VIII) y zona sur (IX, X, XI y XII) con similares proporciones y con una menor concentración en la zona norte (9,9\%: regiones I, II, III y IV).

Desde el punto de vista de su origen, la gran mayoría proviene de Latinoamérica $(83,8 \%)$, mientras que un $16,2 \%$ proviene de los EE.UU., Europa y el resto del mundo. Específicamente, el $28,7 \%$ proviene de Argentina, un 23,1\% de Perú, aproximadamente un 5\% de Brasil, Ecuador, EE.UU., Bolivia, Venezuela y en proporciones aún más bajas, España, Colombia y Alemania (Censo 2002).

En términos generales, la población inmigrante joven en Chile posee un alto nivel de calificación. La mayoría tiene educación secundaria completa y más de un $40 \%$ posee estudios técnicos o universitarios. En relación con el nivel de escolaridad, es posible identificar dos grupos de inmigrantes: uno con bajo nivel de escolaridad y calificación, correspondiente en su mayoría a inmigración de origen intrarregional; y un segundo grupo, con estudios superiores y alta calificación, donde hay europeos, norteamericanos, otras nacionalidades con menor presencia en Chile y también personas de origen intrarregional. Por cierto, estas personas se insertan en trabajos de mayor calidad, con mejores salarios.

En relación a los procesos de integración de la población joven inmigrante en el país, tanto los hombres como las mujeres se ubican principalmente en la categoría de empleado u obrero, seguidos de los trabajadores por cuenta propia, destacando en el caso de las mujeres el alto porcentaje que se dedica a servicios domésticos (cerca de una tercera parte de los casos).

Confirmando lo señalado por otros estudios sobre el tema (Martínez, 2003), el flujo migratorio intrarregional es el único que presenta trabajadoras en la categoría de servicio doméstico. Las mujeres traba-

4 Estos datos representan solo una estimación de la cantidad real de inmigrantes en Chile, pues no considera a las personas migrantes que en el momento de aplicación del instrumento permanecían en calidad de ilegales. 
jan en el sector de servicios domésticos y los hombres en construcción, limitándose con esto las posibilidades laborales percibidas para cada sexo. En esta segmentación del mercado del trabajo confluyen discriminaciones de género, nivel de calificación y país de origen del inmigrante. Estas tres dimensiones determinan la posición social que el individuo ocupará en la sociedad chilena, la cual le reportará mayores o menores oportunidades.

\section{CONSTRUCCIÓN IMAGINARIO SOCIAL DOMINANTE DEL INMIGRANTE POR LA SOCIEDAD CHILENA}

En relación a dichos antecedentes, en una investigación recientemente terminada (Fondecyt $N^{\circ} 1071090$ ), respecto del Ser-Otro inmigrante, concluíamos que si tuviéramos que determinar la presencia de un imaginario social fundacional en Chile, éste tendría que buscarse en la Constitución Política del Estado correspondiente a cada época histórica. Aquella evocará siempre una suerte de «voluntad» de ser chileno plasmada en una perfecta homogeneidad: aquélla que surge de la fusión entre europeos y araucanos, por ejemplo, en la visión del historiador Eyzaguirre (1974). Esta visión ha sido plasmada por los propios inmigrantes europeos por ejemplo en quienes llegaron a colonizar el sur de Chile: «Seremos chilenos honrados y laboriosos como el que más lo fuere, defenderemos a nuestro país adoptivo uniéndonos a las filas de nuestros nuevos compatriotas, contra toda opresión extranjera y con la decisión y firmeza del hombre que defiende a su patria, a su familia y a sus intereses. Nunca tendrá el país que nos adopta por hijos, motivos de arrepentirse de su proceder ilustrado, humano y generoso...» (Anwandter, 1851).

Es desde ese fondo de construcción identitaria que emerge también, en una relación Alter-Ego, la figura del extranjero, aquél que en definitiva personifica la diferencia. En este punto, tal continuidad pone de manifiesto lo que podríamos llamar un «imaginario dominante», entendido en base a la no alteración de la «homogeneidad» étnica inicial y, por ende, más bien restrictivo en sus aperturas hacia flujos migratorios, a excepción de los colonos que son apreciados por su aporte al desarrollo del país.

Los supuestos de dicha investigación se resumen en el hecho de que un imaginario social dominante está siempre en situación de equilibrio precario, por ende, puede haber tanto simples continuidades como inflexiones y cambios. Los GD's efectuados en la misma, en 
materia de Ser-Otro inmigrante, dan cuenta de ambas posibilidades, las cuales pueden ser resumidas en tres aseveraciones.

En primer lugar, destaca la distinción socioimaginaria entre flujos migratorios anteriores, principalmente europeos, y con los cuales se mantiene una relación xenofílica («los alemanes fueron un aporte») $)^{5} \mathrm{y}$, por otra parte, flujos migratorios presentes en la actualidad, principalmente sudamericanos, y con los cuales se genera una relación más bien xenofóbica («argentino, peruano y boliviano... A esos se los discrimina más... Casi a la mayoría pero a esos tres más... Claro y al gringo cero»). ${ }^{6}$

En segundo lugar, se revela casi de forma simultánea la casi inmediata «peruanización» socioimaginaria de la actual inmigración, lo cual imprime una connotación negativa a dicho flujo migratorio («Estamos siendo invadidos por peruanos»). ${ }^{7}$

En tercer lugar, aparece la gradación de la calificación negativa según segmentos socioeconómicos, siendo los sectores de más altos ingresos y de mayor capital cultural quienes demuestran un mayor grado de aceptación no menos jerarquizada socialmente («las nanas peruanas son a menudo personas educadas»). ${ }^{8}$ En relación a ello, y tratándose de una sociedad jerarquizada socialmente, las distancias sociales facilitan la aceptación del Ser-Otro inmigrante, en la medida en que éste no es, por ejemplo, un competidor en el plano laboral. Al revés, las cercanía sociales tienden a ser entendidas como eventualidad de competencia y pugna, en el sentido de que ocuparían puestos de trabajo que deberían ocupar los chilenos («Entonces la ignorancia, no falta... como que les generara un poco de rabia los extranjeros, porque piensan que uno les está robando algo... pero como a un nivel muy bajo»). ${ }^{9}$ Funciona así de manera bastante diferenciada, o bien la actitud favorable y de acogida, o bien la actitud hostil y de rechazo.

En relación a los imaginarios sociales, los entendemos aquí como constituyentes de una realidad social que subyace en el campo de las subjetividades y que conecta a distintas personas a un campo simbólico determinado. «Los imaginarios sociales serían precisamente aquellas representaciones colectivas que rigen los sistemas de identifica-

5 Fondecyt $\mathrm{N}^{\circ}$ 1071090: GD, chilenos, adultos, sector medio, Independencia.

6 INJUV, 2011: GD, inmigrantes jóvenes de Perú y Ecuador, mixto, trabajadores no calificados, Independencia.

7 Fondecyt $\mathrm{N}^{\circ}$ 1071090: GD, chilenos, adultos, sector medio, Independencia.

8 Fondecyt N ${ }^{\circ}$ 1071090: GD, chilenos, adultos, sector Alto, Ñuñoa.

9 INJUV, 2011: GD hombres, argentinos y colombianos, jóvenes profesionales, San Vicente. 
ción y de integración social y que hacen visible la invisibilidad social» (Pintos, 1995:8).

Para Castoriadis, las sociedades existen gracias a la organización de su vida material y la reproducción de la sociedad en sí misma. Para esto el imaginario social y su principal componente social histórico puede ser entendido en el sentido negativo, ya que pone límites a los modos de pensamiento. Sin embargo, a pesar de asumir las limitantes y la carga normativa de un imaginario social, Castoriadis (1975:369) menciona también la posibilidad de nuevos elementos emergentes, un imaginario radical que tiene capacidad creativa y que reafirma al sujeto como un ser vivo (Castoriadis 1975:369). En este sentido, para el autor, los imaginarios sociales tienen una base que tiene la potencialidad de ser imaginario instituyente y a la vez instituido, de manera tal que los mismos pueden ser dinámicos.

\section{CONSTRUCCIÓN DEL INMIGRANTE DESDE EL MUNDO JUVENIL}

En la búsqueda de ese imaginario radical, quisimos indagar sobre las inflexiones al imaginario social dominante interiorizado sobre los extranjeros, especialmente de origen intrarregional y peruanos en la población juvenil chilena, al que nos hemos referido con anterioridad.

De hecho, en la serie de grupos de discusión sobre el tema (Fondecyt $\mathrm{N}^{\circ} 1071090$ ), la juventud presentaba menores niveles de intolerancia y discriminación en particular respecto de los extranjeros, de los peruanos, de las mujeres y de los indígenas en relación a las personas adultas mayores y a los adultos en general. Esta menor discriminación aumentaba con el nivel socioeconómico y el capital cultural y social, y era mayor entre la población de mayor edad, en los sectores de más bajos ingresos y de menor capital cultural.

Así, las inflexiones al imaginario social dominante aparecían de la mano de la juventud, donde las personas jóvenes chilenas eran capaces de distanciarse relativamente de este imaginario social inferiorizante del Ser-Otro inmigrante-peruano, asignándole un valor positivo a dicha inmigración, ya sea por destacar su aporte a la economía del país o a la multiculturalidad del mismo («La inmigración no es molesta, al contrario, ahora Santiago tiene un aire de multiculturalidad que antes no tenía»). ${ }^{10}$

10 Fondecyt No1071090: GD mixto sobre inmigrantes, jóvenes chilenos universitarios, sector alto, Santiago. 
Por ello, es especialmente importante considerar la manera en que la juventud chilena construye al «otro» y las percepciones que tienen de su relación con diferentes grupos sociales. La discriminación - como lo destacábamos en los análisis de la Quinta Encuesta Nacional de Juventud (Aravena, 2007) — es un fenómeno de relaciones intergrupales. Es decir, de relaciones entre diversos grupos sociales. Por eso, resulta interesante observar la percepción que las y los jóvenes chilenos tienen de un conjunto de personas respecto de las que se les consultó a cuáles no les gustaría tener como vecinos (Sexta Encuesta Nacional de Juventud, pregunta 136). ${ }^{11}$ En relación a ello, de diecisiete opciones consultadas en la última Encuesta Nacional de Juventud, los «peruano/bolivianos» ocupan el octavo lugar de rechazo. El rechazo o estigmatización de que gozan los inmigrantes peruanos y bolivianos sin embargo no es menor, situándose en un segundo grupo de «vecinos no deseados».

Sobre el particular, «los neonazis», «los drogadictos», «los flaites» $\mathrm{y}$ «los skinhead» concitan los mayores rechazos por parte de la población juvenil en la sociedad chilena, con un 68\%, 58\%, 47\% y $46 \%$ de rechazo respectivamente, seguidos de los gitanos, homosexuales y lesbianas, personas con problemas de salud mental y peruanos/bolivianos, con un $32 \%, 24 \%, 20 \%$ y $19 \%$, respectivamente; para decaer bajo el $6 \%$ en otras categorías como «musulmanes», «mapuches», «adultos mayores», «pobres», «asiáticos», «personas con discapacidad», «otros extranjeros», «integrantes de otros pueblos indígenas», «otros» $\mathrm{y}$ «ninguno».

Los grupos de personas que los jóvenes prefieren no tener como vecinos varían según el sexo, el nivel socioeconómico, la edad, la localidad de residencia y el nivel educativo alcanzado. Entre los hombres hay mayor rechazo de los peruanos y bolivianos que entre las mujeres. Por nivel socioeconómico, la juventud del segmento más alto tiende a ser más tolerante respecto de los mismos, a diferencia de las personas jóvenes de sectores medios y medios bajos. Esta misma intolerancia es superior en localidades urbanas que en rurales, en jóvenes de menor edad y con menor nivel de educación. A nivel regional, en la Región Metropolitana es donde peruanos/bolivianos concitan el mayor

11 «Pobres», «asiáticos», «musulmanes», «homosexuales/lesbianas», «drogadictos/alcohólicos», «peruanos/bolivianos», «otros extranjeros», «mapuches», «gitanos», «integrantes de otros pueblos indígenas», «adultos mayores», «personas con problemas de salud mental», «neonazis» «skinhead», «personas discapacitadas» «flaites», «otros» $\mathrm{y}$ «ninguno». 
rechazo juvenil, en relación a otras regiones donde la estigmatización de los mismos es inferior, como también es inferior su afluencia.

\section{VIVENCIAS DE LA JUVENTUD MIGRANTE SEGÚN ORIGEN DE LA MIGRACIÓN}

Respecto de las vivencias de la propia juventud inmigrante, los resultados de otro conjunto de grupos de discusión realizados a jóvenes inmigrantes residentes en Chile arrojan que ellos y ellas perciben diferencias de trato y sanciones sociales basadas en su origen extranjero. En efecto, la mayoría percibe un trato hacia los latinoamericanos distinto, peor al trato que se les da a los/as extranjeros/as de origen extrarregional. Dentro del grupo de países latinoamericanos, los más discriminados serían los peruanos, seguidos de los ecuatorianos ( $\ll \mathrm{Si}$ eres europeo o estadounidense eres un Dios aquí, si eres cubano, colombiano vas más o menos, si eres ecuatoriano o peruano $;$ cagaste $\gg){ }^{12}$

Igualmente, las situaciones de discriminación se vivencian en distintos espacios sociales. Uno de ellos es el espacio laboral. En este contexto, la discriminación se expresa en un salario inferior al que recibe una persona chilena; o porque deben demostrar sus habilidades permanentemente; o porque se los somete a una sobrecarga laboral, basados en el supuesto de que se contrata a personas dispuestas a trabajar, sin exigencias («Entre hacerte un contrato de trabajo y no hacértelo... no te lo hacen... te hacen trabajar más de las horas y te pagan sólo lo que se les ocurre pagar»). ${ }^{13}$

Otra diferenciación ocurre en los espacios públicos, principalmente en la vía pública, cuando extranjeros y extranjeras se someten a la mirada de Alter chileno. En estos casos el color de la piel, el fenotipo y el acento, generan un trato desigual. Así, se observa que en nuestro país se tiende a inferiorizar a las personas de color y acento de otros países latinoamericanos ( $\ll \mathrm{A}$ veces uno sufre, las personas te miran feo en la calle... como si fueras de otro planeta... como si fueras un extraterrestre, tú ser moreno, ...para las personas blancas la piel morena no existe»). ${ }^{14}$ De hecho, a más años de residencia en el país y

12 INJUV, 2011: GD, mixto, colombianos, profesionales, Castro.

13 Fondecyt $\mathrm{N}^{\circ} \mathrm{N}^{\mathrm{o}} 1100928$ : entrevista a mujer peruana, joven, sector bajo, Santiago.

14 INJUV, 2011: GD, mujeres, jóvenes, colombianas y ecuatorianas, trabajadoras no calificadas, Puerto Montt. 
a mayor asimilación lingüística, disminuye la percepción de trato negativo. Lo que de acuerdo a los grupos de discusión referidos no sucede lo mismo con el color de la piel («...cuando pasan los más morenos... a veces los insultos y a veces también un hombre pregunta por qué veni acá... ¡vuélvete a tu país, ándate, ándate $)^{15}{ }^{15}$ También se observan otras formas de discriminación. Una de ellas se refiere al tipo de relaciones que establece el Alter chileno con el Ego inmigrante cuando las relaciones se ven mediadas por estereotipos usados por los chilenos para identificar el origen de los jóvenes inmigrantes de ambos sexos. Esta situación es percibida por los/as inmigrantes como ofensiva, puesto que se les trata en relación con la idea que se tiene de ellos y no en relación a cómo ellos se imaginan. Ejemplo de esto es pensar que los hombres peruanos son borrachos («...todos los peruanos son borrachos, ... así he escuchado... en los cinco trabajos que he estado... siempre me dicen oye y a ti te gusta el... copete... el trago,... la droga») ${ }^{16}$ y las mujeres del Caribe son sensuales y guapas, atributo femenino por el cual los hombres consideran a las mujeres en tanto objeto de deseo sexual («Las colombianas que hablan tan rico y no se qué, y la sensualidad... como que andan más despechugadas... como que ese tipo de comentarios me molesta, me molestan de verdad por ese imaginario de mujer tropical más o menos fácil, entonces... ese es un tipo de discriminación recurrente»). ${ }^{17}$ Sobre las percepciones negativas mencionadas, los inmigrantes jóvenes elaboran algunas explicaciones a fin de intentar comprenderlas. Una de ellas dice relación con las imágenes estereotipadas que se transmiten en programas televisivos que buscan destacar las malas condiciones de vida de los inmigrantes en el centro de Santiago, o los problemas legales que se generan con la población indocumentada que cruza las fronteras terrestres del país de manera ilegal («Y no porque una persona se haya comportado mal pueden meter a todos en el mismo saco o generalizar que es lo peor... creo que no tendrían por qué juzgarnos»). ${ }^{18}$

15 INJUV, 2011: GD, mujeres, haitianas, jóvenes, trabajadoras no calificadas, Quilicura.

16 Fondecyt $N^{\circ} 1100928$ : entrevista a hombre peruano, joven, sector bajo, Independencia.

17 INJUV, 2011: GD, mixto, colombianos y paraguayos, estudiantes de postgrado, Santiago.

18 INJUV, 2011: GD mujeres, peruanas, jóvenes, trabajadoras no calificadas, Arica. 
Otra explicación y la más habitual a nivel de sentido común es la mayor competencia en la oferta laboral, producto del aumento de mano de obra extranjera, que afecta a ciertos sectores de la economía. Especialmente en el caso de la percepción de las mujeres extranjeras que trabajan en el servicio doméstico, es que deben competir con las mujeres chilenas que trabajan en dicho rubro. Así, la competencia como elemento no deseable serviría para explicar el trato discriminatorio que reciben algunas mujeres ( «Los chilenos dicen que los peruanos vienen y les quitan el trabajo, pero yo siempre les digo... el peruano se lo gana... el peruano acá no viene a robar un puesto de trabajo a un chileno, viene a ganárselo»). ${ }^{19}$ Esta percepción ha sido validada por nuestra investigación sobre Imaginarios Sociales del Otro en el Chile Contemporáneo (2007-2010), ${ }^{20}$ en la que se constató, en primer lugar, que la inmigración se peruanizaba en grupos de discusión de chilenos sobre los extranjeros y que en los segmentos socioeconómicos chilenos más bajos y de menor capital cultural existía una actitud de mayor discriminación hacia alter peruano.

De hecho esa es una de las razones indicadas por Ego inmigrante para explicar el trato desigual hacia este grupo, dando cuenta de la falta de educación, o simplemente, la ignorancia de Alter chileno como la base de prácticas de maltrato o discriminatorias hacia el colectivo de inmigrantes («Depende de la mentalidad que tenga la persona... con la cual va a hablar...»). ${ }^{21}$

Por último, hay un grupo de jóvenes inmigrantes que declaran no haber vivido situaciones de discriminación. Basados en las relaciones de buen trato y vínculos de confianza que algunos de ellos y ellas establecen con las personas chilenas primaría un sentimiento de integración social, que les impediría percibir otras situaciones de discriminación o criticar ciertos tratos diferenciados, basados en su origen foráneo («El trato dependería de qué país vienes... todos piensan aquí que soy de un buen colegio y es como ridículo porque soy canadiense. Yo veo racismo en Chile y discriminación pero para mí obviamente... en este caso yo no puedo quejarme, es lamentable»). ${ }^{22}$

19 INJUV, 2011: GD mujeres, peruanas, jóvenes, trabajadoras no calificadas, Arica.

20 Fondecyt $\mathrm{N}^{\circ} 1071090$.

21 Fondecyt $\mathrm{N}^{\mathrm{o}}$ 1100928: entrevista, hombre, peruano, joven, estudiante secundario, Independencia.

22 INJUV, 2011: GD hombres, jóvenes, de inmigración extrarregional, profesionales, Talca. 


\section{A MODO DE CONCLUSIÓN}

La multiplicidad de aristas de la temática nos ha llevado a abordar el mismo desde una triple perspectiva. La primera, ha considerado una caracterización de la migración juvenil en Chile; la segunda, la construcción imaginario social dominante del inmigrante por la sociedad chilena; y la tercera, la vivencia de la migración por parte de jóvenes inmigrantes en Chile y su relación con la sociedad chilena contemporánea.

Sobre la primera perspectiva hemos podido establecer que sin duda la afluencia de flujos migratorios juveniles hacia Chile ha sido muy importante en las últimas décadas, especialmente de acuerdo a la información que arroja el último Censo y las encuestas Casen. Al respecto, si bien esta migración es bastante heterogénea, existen diferencias considerables respecto de flujos migratorios intrarregionales que han llegado a desempeñarse como mano de obra de menor calificación, en relación a flujos extrarregionales que han llegado en calidad de estudiantes, de turistas o de ejecutivos de empresas internacionales.

En relación a la segunda perspectiva, Pintos (1995:5) sostiene que hay aristas trascendentales en la constitución del imaginario dominante; uno de los principales es el determinismo histórico y la autoridad que tiene éste en la supresión de sujetos individuales y colectivos. Como hemos visto en los resultados expuestos, ello se expresaría en la invisibilización de las personas jóvenes de origen extranjero, especialmente intrarregional, y su reemplazo por estereotipos construidos sobre ellos. Señala también una serie de elementos que funcionan a favor del imaginario dominante, el primero de ellos es el restablecimiento del darwinismo social, en el sentido de presentar los hechos sociales como si estuvieran sujetos a leyes naturales, respecto de lo cual hemos constatado la manera en que se tiende a naturalizar el carácter negativo de aquellos inmigrantes que son percibidos también de manera negativa. El autor también menciona la ruptura del ámbito público y privado como consecuencia del creciente sometimiento al mercado. En efecto, se evidencia que en los casos estudiados habría una mejor valoración del inmigrante cuando éste se desenvuelve en actividades económicas valoradas para la población nacional, y; por último, expone la importancia que ha tenido la globalización en términos de comunicación de masas, donde los imaginarios se renuevan y tienen anclaje a los medios y la tecnología. En el caso de este trabajo, se puede destacar la influencia de las imágenes mediatizadas sobre la 
vida y condiciones socioeconómicas en que se desenvuelven ciertos grupos de inmigrantes, como los peruanos en Chile, trascendiendo una imagen negativa para el conjunto de la población del mismo origen.

Respecto a la tercera perspectiva, debemos entender que los imaginarios sociales funcionan como constructores de realidad social, dotan de sentido a la sociedad y se mueven en el universo simbólico. Para Castoriadis, las instituciones y las significaciones imaginarias permiten crear un mundo dotado de sentido (Castoriadis, 1997:5). La institución de la sociedad es la que decide qué es real y qué no lo es, con su propio esquema de interpretación del mundo donde lo socialhistórico tendrá especial relevancia. Así, podemos concluir que efectivamente existe una mayor discriminación de los inmigrantes jóvenes latinoamericanos en Chile, especialmente si son peruanos. Que ello no sólo se desprende de la percepción de los propios jóvenes inmigrantes, sino que es confirmado por chilenos y chilenas en grupos de discusión. También es sostenido por jóvenes chilenos a la luz de los resultados de las Encuestas Nacionales de Juventud.

No obstante ello, en todos los casos, las diferencias respecto de la aceptación/rechazo, tolerancia/intolerancia, vendrían de la mano del capital cultural y de los niveles de educación de la población chilena que pertenece a los grupos de también mayor capital social. Igualmente, se observa una mayor aceptación del inmigrante origen intrarregional en las personas más jóvenes de nuestra sociedad. Ello da cuenta de la posibilidad de que surjan cambios al imaginario dominante de acuerdo a las discontinuidades planteadas por Castoriadis. Las mismas han sido observadas en las inflexiones que comienzan a aparecer en los segmentos etarios más jóvenes en el Chile contemporáneo. Las personas jóvenes demuestran así una mayor capacidad creativa respecto de los estereotipos construidos, contribuyendo a la configuración de nuevos imaginarios sociales.

SANTIAGO (CHILE), ABRIL 2012

RECIBIDO, ABRIL 2012

ACEPTADO, MAYO 2012 


\section{REFERENCIAS BIBLIOGRÁFICAS}

ANWANDTER, CARLOS (1851): Disponible en: www.scribd.com.

ARAVENA, ANDREA (2007): «Discriminación e intolerancia». Quinta Encuesta Nacional de Juventud. Santiago: INJUV.

CASTORIAdis, CoRnelius (1997): El imaginario social instituyente. Buenos Aires: Eudeba. (1975): La institución imaginaria de la sociedad. Barcelona: Tusquest.

EYZAGUIRRE, JAIME (1974): Historia de Chile. Santiago: Zig-Zag.

FONDECYT N¹071090: «Imaginarios sociales del otro en el Chile contemporáneo: la mujer, el indígena y el inmigrante». Investigadores: Manuel Baeza, Andrea Aravena y Miguel Urrutia. Concepción: Universidad de Concepción.

FONDECYT N¹100928: «Vivencia de imaginarios sociales en víctimas de violencia simbólica en Chile». Investigadores: Manuel Baeza, Andrea Aravena y Bernardo Castro. Concepción: Universidad de Concepción.

InSTITUTO NACIONAL DE EstadístICA (INE) (2002): «Base de datos XVII censo nacional de población y VI de vivienda, 2002». Santiago: INE.

INSTITUTO NACIONAL DE LA JUVENTUD (INJUV) (2011): Jóvenes migrantes: inclusión social y desafíos para las políticas publicas en juventud. Santiago: INJUV.

(2010): Sexta encuesta nacional de juventud. Santiago: INJUV. (2007): Quinta encuesta nacional de juventud. Santiago: INJUV.

MARTíneZ, JORGE (2003): «El encanto de los datos. Sociodemográfica de la inmigración en Chile según el censo 2002». Serie Población y Desarrollo No49. Santiago: CELADE.

Ministerio DE PLANIFICACIÓN Y DESARROLlO (2006): Encuesta de caracterización socioeconómica nacional, CASEN 2006: Santiago: MIDEPLAN.

PINTOS, JUAN LUIS (1995): Los imaginarios sociales. La nueva construcción de la realidad. Madrid: Fe y Secularidad. 